\title{
Climbing a long hill: pelvic floor surgery and the need for geriatric urogynecology
}

\author{
Cornelia Betschart • Diaa E. E. Rizk
}

Received: 12 November 2013 / Accepted: 6 December 2013 / Published online: 23 January 2014

(C) The International Urogynecological Association 2014

The topic of geriatric urogynecology is receiving much attention lately as a public health priority because of the global increase in female life expectancy and the significant risk of associated pelvic floor dysfunctions. Statistics from the USA project that in $2050,25 \%$ of the population will be $>60$ years and that those $>85$ will increase from a population of $1.9 \%$ in 2010 to $4.3 \%$ in 2050 [1]. The urogynecological community should thus expect to face a worldwide and progressively growing demand from patients seeking care for pelvic floor dysfunctions. One study predicted $>50 \%$ increased demand for urogynecological services over the next 40 years [2].

The changing female demographics is accompanied by a considerable interest in the contemporary literature to standardize the indications, techniques, and outcomes of surgical treatment of pelvic floor dysfunctions. Only a few reports appreciate that increasing age of our female population is an intrinsic biological variable interfering with the standardization process [3]. Women aged $>80$ years undergoing urogynecological surgery have a 13.6 higher risk of postoperative death than their younger counterparts. In the majority of cases, the cause of death is not directly related to the procedure [4]. Despite the obvious need for operative intervention among these elderly women and the strong evidence for the inherent morbidity and mortality of this treatment option,

\section{Betschart $(\bowtie)$}

Department of Gynecology, University Hospital Zurich,

Frauenklinikstrasse 10, 8091 Zürich, Switzerland

e-mail: cornelia.betschart@gmx.ch

\section{E. E. Rizk}

Department of Obstetrics and Gynecology, Faculty of Medicine, Ain Shams University, Cairo, Egypt

e-mail: rizk.diaa@gmail.com

\section{E. E. Rizk}

The Canadian Continence Foundation, 671 Elgin Street, Peterborough, Ontario K9H 2E2, Canada there is scarce urogynecological data on the risks and specific perioperative management in this patient group. Ageing influences lower urinary tract symptoms (LUTS), pelvic floor function, and perioperative and postoperative courses. The higher postoperative morbidity rate is also an important factor that is often overlooked in most surgical studies. Main concerns when treating elderly women are:

(1) Functional aging with myogenic and neurological changes

(2) Polypharmacy

(3) Impaired cognitive function and risk of delirium

First, considering functional aging, it is traditionally believed that aging is simply a physiological phenomenon caused by a gradual and exponential decline in the functional reserve. Aging, however, is a complex and evolutionary spectrum of innate and progressive events affecting molecules, cells, and the entire organism and is caused by free radicals, nonenzymatic glycosylation, and apoptosis [5]. The entire process is under the control of the endocrine systems - mainly growth hormone, estrogens, and androgens - with significant acceleration of the pace of ageing in women than in men [5]. Aging induces ultrastructural alterations in the urinary bladder that include apoptosis of the detrusor muscle, widening of the intercellular tight junctions, and degeneration of the axonal innervation [6]. These lesions may contribute to the development of functional disorders such as higher postvoid residual volumes, postoperatively impaired voiding dysfunction, or sensory micturition disturbances. The mechanism by which aging changes in the pelvic organs, particularly the urinary bladder, can influence the pathogenesis of LUTS; however, is not fully elucidated. Elderly people are more likely to suffer from metabolic diseases such as diabetes mellitus, hypothyroidism, renal insufficiency, and malnutrition, which may aggravate preoperative LUTS 
via an exaggerated metabolic response to surgical trauma [3].

Second, polypharmacy common in the elderly population can adversely affect operative outcome and postoperative recovery. Common medications include antiplatelet therapy (APT), which may interfere with operative hemostasis; and drugs with anticholinergic side effects, such as antidepressants and neuroleptics, which may induce postoperative delirium, cognitive impairment, or voiding dysfunction. The decision to discontinue the use of APT before urogynecological surgery depends on the original preoperative indication [7]. According to the American College of Chest Physicians, the use of prophylactic APT is subdivided into primary indications that are recommended in high-risk groups (categorized by the Framingham Score) and secondary indications that comprise artificial heart valves, stents, acute coronary syndrome, ischemic stroke, and peripheral artery disease. Premature withdrawal of APT is associated with a significant higher risk of ischemic events. The balance of risk between hemorrhage versus thrombosis, therefore, should be carefully weighed by the urogynecologist operating on elderly women. In some women, it may be better to postpone operative intervention until the patient has a more stable cardiovascular function. The incidence of venous thromboembolism also increases with age [8]. In general, urogynecological operations are associated with a moderate risk of thromboembolism. Hence, if no other risk factors are present, there is no need for prolonged antithrombotic therapy after the patient's discharge. Age and its sequelae are clearly mirrored in the American Society of Anesthesiologists (ASA) score, which increases significantly with patient age. Additional factors increasing surgical morbidity include longer operation time, excessive blood loss, and general anesthesia.

Third, cognitive impairment and the use of benzodiazepines increases with age, as does the risk for falls and fractures $[8,9]$. Cognitively impaired elderly patients often develop postoperative delirium, which is associated with increased mortality, falls, and respiratory complications such as pneumonia. Delirium also increases the possibility of discharge from hospital to long-term nursing homes and further cognitive decline in the following years [10]. To prevent postoperative delirium in susceptible patients, preoperative preparation should include strict adherence to the night-and-day sleep cycle; hospital admission to closely monitor the cognitive state; assessment of blood electrolyte balance, especially serum sodium levels; and avoiding the use of anticholinergic drugs or drugs with anticholinergic side effects.

In our opinion, the vaginal route is preferable for pelvic reconstructive surgery in elderly women, as it usually takes shorter operating time and can be performed under regional anesthesia. Obliterative vaginal procedures are an effective option in elderly patients who cannot tolerate extensive surgery and who are not planning future sexual activity.
In conclusion, biological age should not deter surgical treatment for women with pelvic floor dysfunctions. However, the risk of postoperative complications is definitely increased in elderly women undergoing urogynecological surgery. A proactive strategy is urgently needed to minimize postoperative morbidity and mortality and optimize operative outcomes and elucidate the interdependent role of anesthesia. The key components are a multidisciplinary approach to preoperative assessment in collaboration with physicians and anesthesiologists, and greater awareness of the pathophysiology of aging and the subsequent potential for postoperative complications. Until more information is available on this subject, elderly women with pelvic floor dysfunctions should be the target population in future surgical trials; this subgroup had been too often and unfairly excluded from enrollment. We firmly believe that geriatric urogynecology is here to stay and will rapidly expand in the next decade based on the logical wisdom that "necessity is the mother of invention."

Conflicts of interest None.

\section{References}

1. Department of Health and Human Services, Administration on Aging. Aging statistics. Available at: http://www.aoa.gov/aoaroot/ aging_statistics/index.aspx. Accessed August 20, 2013

2. Wu JM, Hundley AF, Fulton RG, Myers ER (2009) Forecasting the prevalence of pelvic floor disorders in US women: 2010 to 2050. Obstet Gynecol 114:1278-1283

3. DuBeau CE, Kuchel GA et al (2010) Incontinence in the frail elderly: Report from the 4th International consultation on Incontinence. Neurourol Urodynam 29:165-178

4. Sung VW, Weitzen S, Sokol ER, Rardin CR, Myers DL (2006) Effect of patient age on increasing morbidity and mortality following urogynecologic surgery. Am J Obstet Gynecol 194:1411-1417

5. Rizk DEE, Fahim MA (2008) Ageing of the female pelvic floor: Towards treatment "a la carte" of the "geripause". Int Urogynecol J $19: 455-458$

6. Elbadawi A, Yalla SV, Resnick NM (1993) Structural basis of geriatric voiding dysfunction. II. Aging detrusor: normal versus impaired contractility. J Urol 150(5 Pt 2):1657-1667

7. Vandvik PO, Lincoff MA, Gore JM, Gutterman DD, Sonnenberg FA, Alonso-Coello P et al (2019) Prevention of Cardiovascular Disease: Antithrombotic Therapy and Prevention of Thrombosis, 9th ed: American College of Chest Physicians Evidence-Based Clinical Practice. Guidelines Chest 141(2 suppl):e637S-e668S

8. Lakatos BE, Capasso V, Mitchell MT, Kilroy SM, Lussier-Cushing M, Sumner L, Repper-Delisi J, Kelleher EP, Delisle LA, Cruz C, Stern TA (2009) Falls in the general hospital: association with delirium, advanced age, and specific surgical procedures. Psychosomatics 50(3):218-226

9. Pariente A, Dartigues JF, Benichou J, Letenneur L, Moore N, Fourrier-Réglat A (2008) Benzodiazepines and injurious falls in community dwelling elders. Drugs Aging 25(1):61-70

10. Marcantonio ER, Goldman L, Mangione CM et al (1994) A clinical prediction rule for delirium after elective noncardiac surgery. JAMA 271:134-139 